\title{
Associations between health status and the probability of approaching a novel object or stationary human in preweaned group-housed dairy calves
}

\author{
M. C. Cramer ${ }^{1}$ and A. L. Stanton \\ Department of Dairy Science, University of Wisconsin, Madison 53706
}

\begin{abstract}
Neonatal calf diarrhea (NCD) and bovine respiratory disease (BRD) are the 2 most prevalent diseases affecting the welfare and productivity of preweaned dairy calves in the United States. Early detection of these diseases improves both the probability of recovery and animal welfare. Group housing of preweaned calves is increasing in popularity and disease detection in a socially competitive environment can be challenging. One method of detecting disease is through the observation of key behaviors that occur during illness. Novel object and stationary human approach tests can be used to measure exploratory behavior, which is decreased during the expression of sickness behavior. The objectives of this study were (1) to determine associations between 4 categories of health status and the probability of calves approaching a novel object or stationary human, and (2) to determine the associations between rectal temperature and the probability of calves approaching. Holstein heifer calves $(\mathrm{n}=75)$ in group housing were tested weekly for the first $6 \mathrm{wk}$ of life for their willingness to approach a novel object (OBJ) or stationary human (SH). After the approach tests, calves were evaluated by research staff using a standardized health-scoring system. Treatment records were obtained from the farm to determine detection of BRD, NCD, and umbilical infections. Associations between probability of approach and BRD status were analyzed using a linear mixed model with a logittransform (PROC GLIMMIX), controlling for calf as a random effect. All models controlled for week, pen, pen order, and test order. Calves with clinical signs of BRD on test day were 0.5 [95\% confidence interval (CI) $=0.3-0.9]$ and $0.5(95 \% \mathrm{CI}=0.3-0.9)$ times as likely to approach the OBJ and SH compared with healthy calves, respectively. Calves with a fever (rectal temperature $\left.\geq 39.4^{\circ} \mathrm{C}\right)$ were $0.4(95 \% \mathrm{CI}=0.2-0.8)$ and 0.4
\end{abstract}

Received March 4, 2015.

Accepted June 21, 2015.

${ }^{1}$ Corresponding author: mccramer@wisc.edu
$(95 \% \mathrm{CI}=0.1-0.7)$ times as likely to approach the OBJ and SH compared with calves without fever, respectively. Calves that were recovering from NCD on test day, but were not showing clinical signs of NCD, were $0.4(95 \% \mathrm{CI}=0.2-0.9)$ times as likely to approach $\mathrm{SH}$, compared with healthy calves. These results suggest that approach tests may be useful for the identification of calves with clinical signs of BRD and calves with a fever in group housing.

Key words: calf, behavior, bovine respiratory disease, neonatal calf diarrhea, welfare

\section{INTRODUCTION}

Neonatal calf diarrhea (NCD) and bovine respiratory disease (BRD) are the 2 most prevalent diseases affecting preweaned dairy calves in the United States. According to producer-reported information, NCD and BRD affect 23.9 and $12.4 \%$ of preweaned calves in the United States, respectively (USDA, 2010). Producers cited NCD and BRD as the causes for 56.5 and $22.5 \%$ of preweaned heifer deaths, respectively (USDA, 2010).

Neonatal calf diarrhea has been associated with BRD (Hultgren et al., 2008), an increased age at first calving (Waltner-Toews et al., 1986), reduced ADG (Windeyer et al., 2014), and may be painful (Todd et al., 2010). Bovine respiratory disease negatively affects the economic viability of a dairy and is associated with reduced growth (Virtala et al., 1996a; Stanton et al., 2012), increased likelihood of mortality (WaltnerToews et al., 1986; Stanton et al., 2012), increased age at first calving (Waltner-Toews et al., 1986; Stanton et al., 2012), decreased calving ease (Stanton et al., 2012), and an increased risk of not completing the first lactation (Bach, 2011). Bovine respiratory disease and NCD may be accompanied by feelings of malaise, which can reduce animal welfare (Millman, 2007).

To minimize these consequences, methods to accurately and efficiently identify ill calves are needed. Producers are relatively capable of correctly identifying NCD or BRD, but have been shown to have poor sensitivity when considering overall illness identification (Sivula et al., 1996). In a cross-sectional study, farm 
treatment records were compared with results from standardized health scores; the sensitivity of farm for identification of BRD was $24 \%$, which was calculated as the proportion of calves identified by research staff with BRD that were treated by the farm in the previous $7 \mathrm{~d}$ (M. C. Cramer and A. L. Stanton, unpublished data). This presents a challenge, as late detection of illness can reduce treatment success and increase the rate of recurrence (McGuirk, 2008).

Currently, several methods of disease identification exist, including examination of clinical signs of individual animals (McGuirk, 2008), detecting changes in feeding behavior data from automated feeders (Svensson and Jensen, 2007; Borderas et al., 2009), radio frequency technology (Quimby et al., 2001), and noninvasive infrared thermography (Schaefer et al., 2012). Whereas these methods represent options for disease detection, daily individual health exams may be timeconsuming and the automated monitoring technologies may not be financially feasible for all farms. Therefore, alternative methods of disease detection are needed.

The observation of calf behavior may facilitate detection of disease. Sickness behavior is a term that is used to describe characteristic changes in behavior that occur as a result of physiologic changes associated with illness. The behavioral changes may include lethargy, increased lying time, isolation from other animals, and decreased grooming, appetite, exploratory behavior, and interest in novelty (Hart, 1988; Haba et al., 2012). Observation of these behavioral changes represents an opportunity for a low-cost method of disease detection.

Object and human approach tests have been used in adult cattle to examine temperament and fear responses (e.g., Gibbons et al., 2009) and in heifer calves from 2 to 6 wk of age (Lauber et al., 2006). However, these were performed in an unfamiliar environment and the effect may be different when performed in a familiar environment because fear and exploratory behavior can be competing motivations (Murphy and Wood-Gush, 1978). Exploratory behavior toward a novel object is decreased when mice are injected with LPS, which induces sickness behavior (Haba et al., 2012). Therefore, a modified approach test may provide a potential method to measure changes in exploratory behavior in preweaned dairy calves that are due to illness. To our knowledge, the relationship between approach tests and illness has not been studied in cattle. Thus, the objectives of the current study were to (1) determine the associations between 4 categories of health status on the probability of approaching a novel object or stationary human and (2) to determine the associations between rectal temperature on the probability of approaching and a novel object or stationary human.

\section{MATERIALS AND METHODS}

\section{Animals and Housing}

The Animal Care and Use Committee at the University of Wisconsin-Madison approved all procedures in our study (protocol A01516-0-09-12). The study was conducted on a commercial dairy (consisting of 2 source farms) containing 3,700 lactating cows in Wisconsin. Holstein heifer calves $(\mathrm{n}=79)$ were enrolled upon entry into 1 of 8 pens where they were housed in stable groups of 8 to 10 calves throughout the trial. No random assignment to pen was carried out because the farm filled each pen as calves were born. Calves were enrolled in 2 cohorts over a period of $2 \mathrm{wk}$. Calves entered the pens between one and $2 \mathrm{~d}$ of age and were monitored for 6 wk. Each pen measured $3.7 \times 3.8 \mathrm{~m}$ and contained 1 water dish and 2 nipple feeders. The distance between the 2 nipples was $75 \mathrm{~cm}$. Pen flooring consisted of slats covered with a rubberized material (Cozy Calf Cribs, Blue Diamond Industries LLC., Strawberry Point, IA). The back portion $(3.7 \times 2.6 \mathrm{~m})$ of the pen had flooring with supplemental heat whereby hot water ran through steel pipes attached to the bottom of the flooring system. Calves were offered acidified waste milk ad libitum; milk was acidified to a $\mathrm{pH}$ of 4.0 to 4.5 using formic acid, reheated to 21 to $38^{\circ} \mathrm{C}$, and pumped from the milk reservoir through plastic tubing to nipples in the pens, which contained one-way valves based on the method of Anderson (2013). Calves were weaned when calves in the pen averaged 6 wk of age. The farm weaned calves over a period of $5 \mathrm{~d}$ by decreasing the amount of time milk was available. No milk intake data were available for this system.

\section{Data Collection}

Weekly farm visits by research staff consisted of administering approach tests followed by a standardized health score, height (Calf height stick, Nasco, Fort Atkinson, WI), weight (Holstein dairy calf weigh tape, Nasco), and a blood sample via jugular venipuncture for a separate study. Enrollment of calves took place over 2 wk with 52 and 27 calves enrolled during the first and second farm visit, respectively. Research staff observed calves weekly for the first 6 wk of life. Test day refers to the day that research staff visited the farm for health assessments and approach tests.

Behavioral Assessments. All approach tests were performed before health examinations. Two approach tests were used: novel object (OBJ) and stationary human (SH). For each test, calves were given $60 \mathrm{~s}$ to approach. For OBJ, a single researcher entered each pen 
Table 1. Standardized health-scoring system

\begin{tabular}{|c|c|c|c|c|}
\hline Score & 0 & 1 & 2 & 3 \\
\hline Rectal temperature $\left({ }^{\circ} \mathrm{C}\right)$ & $37.8-38.2$ & $38.3-38.8$ & $38.9-39.3$ & $\geq 39.4$ \\
\hline Nasal discharge & Normal serous discharge & $\begin{array}{l}\text { Small amount of } \\
\text { unilateral cloudy } \\
\text { discharge }\end{array}$ & $\begin{array}{l}\text { Bilateral, cloudy, or } \\
\text { excessive mucous } \\
\text { discharge }\end{array}$ & $\begin{array}{l}\text { Copious bilateral } \\
\text { mucopurulent discharge }\end{array}$ \\
\hline Ears & Normal & Ear flick or head shake & Slight unilateral droop & $\begin{array}{l}\text { Head tilt or bilateral } \\
\text { droop }\end{array}$ \\
\hline Neonatal calf diarrhea score & $\begin{array}{l}\text { Normal: Fecal consistency } \\
\text { thicker that orange juice }\end{array}$ & $\begin{array}{l}\text { Diarrhea: Feces } \\
\text { extremely watery; } \\
\text { consistency of orange } \\
\text { juice }\end{array}$ & & \\
\hline Umbilical score & $\begin{array}{l}\text { Cord cannot be found, } \\
\text { umbilical opening is } \\
\text { completely flush with the } \\
\text { abdomen of the calf }\end{array}$ & $\begin{array}{l}\text { Cord can be palpated, } \\
\text { but is soft and } \\
\text { approximately the size } \\
\text { of a pencil }\end{array}$ & $\begin{array}{l}\text { Cord is larger than } \\
\text { a pencil, but the calf } \\
\text { does not react when } \\
\text { palpated }\end{array}$ & $\begin{array}{l}\text { Umbilical is very } \\
\text { swollen, hard and hot } \\
\text { to the touch, and calf } \\
\text { reacts when palpated }\end{array}$ \\
\hline
\end{tabular}

${ }^{1} \mathrm{BRD}=$ bovine respiratory disease; scoring adapted from McGuirk (2008).

and placed a blue polyurethane cone that measured $21.4 \mathrm{~cm}$ tall and $14 \mathrm{~cm}$ at the base (Nasco) in the center of the pen, and then left the pen for $60 \mathrm{~s}$. For SH, the researcher calmly walked to the center of the pen and remained motionless for $60 \mathrm{~s}$. Pen order (1-8) and test order (OBJ or $\mathrm{SH}$ ) were randomized each week. All approach tests were conducted between 1000 and 1200 $\mathrm{h}$ on the same day every week.

Health Assessments. Following the approach tests, all calves $(\mathrm{n}=79)$ underwent standardized health assessments by trained research staff (Table 1). Calves were scored for BRD using a BRD screening tool (McGuirk, 2008). Fecal consistency was observed if the calf defecated or if feces were present on the perineal area of the calf. As part of the health assessment, a rectal temperature was taken on every calf, which provided a mild stimulant of defecation. The umbilicus of every calf was palpated and categorized using a 4-point scale.

\section{Disease Identification by Farm Staff}

In addition to weekly health assessments performed by research staff, farm staff monitored calves daily for signs of illness. If a calf was determined to be sick by farm staff, calves were treated according to standardized farm treatment protocols. The farm staff treated calves for respiratory disease when they were older than 2 wk of age, had a rough hair coat, had wheezing in lungs identified by auscultation with a stethoscope, and had a rectal temperature of $39.4^{\circ} \mathrm{C}$ or greater, or if an ear droop or head tilt was present. They treated calves for NCD when the calf was observed with watery feces. Calves were treated for umbilical infections when the umbilicus was swollen, tender, or abnormal discharge was observed. Farm staff recorded treatment records in Excel (Microsoft, Redmond, WA) and these were collected by research staff weekly. Treatment records were used to determine cases of illness not identified by research staff. For ethical reasons, the results of the health scores were emailed to the farm owner $24 \mathrm{~h}$ after the health scores and approach tests were performed.

\section{Disease Definitions}

Calves were categorized as having clinical signs of NCD on test day or recovering from NCD. If fecal consistency for a calf on a test day was extremely watery (consistency of orange juice) and identified by research staff, it was considered clinical NCD (DIACLIN; Table 1). The recovery period of NCD was defined as $7 \mathrm{~d}$ from last identification by either farm or research staff. As such, a calf recovering from NCD (DIAREC) was defined as a calf that showed signs of NCD in the previous $7 \mathrm{~d}$ but did not show clinical signs on test day (McGuirk, 2008). A case of NCD was considered resolved when the calf was not identified with NCD in the previous $7 \mathrm{~d}$; after this time, identification of the calf with NCD would be considered a new case.

An overall BRD score was obtained by totaling the scores for rectal temperature, cough, ears, and the highest score between the nasal and eye score (Table 1). Calves were categorized as having clinical signs of BRD on test day or as recovering from BRD. Clinical BRD on test day was defined as at least 2 abnormal (score of 2 or greater) clinical signs as identified by research staff (BRDCLIN). The recovery period of BRD was defined as $10 \mathrm{~d}$ from last identification by either farm or research staff. As such, a calf recovering from BRD 
(BRDREC) was defined as a calf that showed signs of $\mathrm{BRD}$ in the previous $10 \mathrm{~d}$ but did not show clinical signs on test day (McGuirk, 2008). A case of BRD was considered resolved when a calf was not identified with BRD in the previous $10 \mathrm{~d}$; after this time, identification of the calf with BRD would be considered a new case.

Rectal temperatures (TEMP) were assigned a score from 0 to 3 as part of the BRD screening tool (Table 1). A score of 3 was considered febrile. A score of 3 for umbilical health was considered infected (Table 1). A case was considered resolved when the calf had an umbilical score of less than 3. After the farm visit, a list of any sick calves identified by research staff was given to farm staff.

\section{Video Observation of Approach Tests}

All tests were recorded using digital cameras (Panasonic color CCTV, MV-CP484, Osaka, Japan) installed before the study; one camera was installed per pen. Individual coat patterns and markings were used to identify calves on video. A single observer blind to health status conducted all video observations. For OBJ, the start time was recorded as the moment when the object was placed in the pen. For SH, the start time was recorded when the researcher was in the pen and standing completely motionless. Approach was defined as 1 step in the direction of the OBJ or SH such that the distance between the 2 decreased, within $60 \mathrm{~s}$ of test initiation. The decision to only use 1 step was based on the smaller pen size and the number of calves, and this allowed us to demonstrate willingness to approach. During video observations, it was considered 1 step only if it was voluntary, if a calf was bumped by another calf, this was not recorded as an approach.

\section{Sensitivity, Specificity, and Predictive Values}

Sensitivity (Se), specificity $(\mathbf{S p})$, predictive value positive $(\mathbf{P V}+)$, and predictive value negative $(\mathbf{P V}-)$ of the approach tests were calculated as described by Dohoo et al. (2003), and calculations represent all weeks combined unless otherwise noted. A positive test was defined as not approaching either OBJ or SH. Sensitivity is the proportion of sick calves that test positive and $\mathrm{Sp}$ is the proportion of healthy calves that test negative. Predictive value positive is defined as the probability that calves that test positive truly are sick. Predictive value negative is defined as the probability that calves that test negative are truly healthy. The calculations for test characteristics (Se, Sp, PV+, and $\mathrm{PV}-$ ) were only performed for health status variables that were significantly associated with the probability of approaching OBJ and SH. Calves were considered healthy if they did not have BRDCLIN, BRDREC, DIACLIN, DIAREC, or a TEMP score of 3. This definition of healthy was used for all Se and Sp calculations. The Se of farm staff for disease identification was defined as the proportion of disease cases (BRDREC) that were treated by the farm staff during the duration of the case.

\section{Statistical Analysis}

The calf was the experimental unit. Data were analyzed using SAS statistical software (SAS Institute Inc., Cary, NC). Results of the approach tests (OBJ and SH) were analyzed separately. Health status and rectal temperature were analyzed separately to determine their association with approach. Fever is frequently a subset of disease, particularly of BRD, and separate models allowed for examination of a single clinical sign without confounding.

Initial screening of variables was performed to determine which variables were necessary to include in the multivariable model. Chi-squared analyses were performed at the univariable level using PROC FREQ (SAS Institute Inc.) to determine associations between approach tests and the following variables: treatment history (if the calf was treated in the previous $3 \mathrm{~d}$ ), pen, order in which the pen was tested, enrollment cohort, test order, farm, and week (age of the calf in weeks). Chi-squared analyses that resulted in $P \leq 0.20$ were included in multivariable analysis. Multivariable models were constructed using stepwise backward removal of variables and all final models included pen, order in which the pen was tested, enrollment cohort, test order, farm, and week (age of the calf in weeks). A summary of final models and variable definitions can be found in Table 2. Statistical significance was defined as $P<$ 0.05 .

The probability of approach was analyzed using a linear mixed model (PROC GLIMMIX, SAS) with a logit-transform and calf was controlled for as a random effect. Odds ratios were then calculated based on the probability of approach from these models. The covariance structure was autoregressive and was selected based on the lowest Akaike's information criterion.

\section{RESULTS}

Five calves were not tested during wk 1 because they were enrolled after the pen-level approach tests were complete; these calves were only behavior tested for 5 wk. One pen of calves $(\mathrm{n}=9)$ was inadvertently skipped during the approach tests in wk 2 . A few calves were temporarily removed from the study pen during wk $2(\mathrm{n}=1), 3(\mathrm{n}=1)$, and $6(\mathrm{n}=1)$, resulting in 
Table 2. Summary of variables in statistical models

\begin{tabular}{llll}
\hline Model & Variable & Range & Definition \\
\hline Disease & Pen & $1-8$ & The 8 pens tested \\
& Pen order & $1-8$ & Order in which each pen was tested \\
& Enrollment cohort & 1 or 2 & Cohort in which each calf was enrolled in the study \\
& Test order & 1 or 2 & Order in which each test (novel object or stationary human) was performed \\
& Farm & 1 or 2 & Farm of origin \\
& Week & $1-6$ & Age of the calf, in weeks \\
& BRDCLIN & Yes or No & Clinical signs of BRD ${ }^{1}$ were identified by research staff; BRD defined as at least 2 \\
& BRDREC & Yes or No & Signs of BRD in previous 10 d; no clinical signs on test day \\
& DIACLIN & Yes or No & Clinical signs of neonatal calf diarrhea on test day were identified by research staff \\
& DIAREC & Yes or No & Signs of neonatal calf diarrhea (extremely watery feces) in previous 7 d; no clinical \\
& Pen & $1-8$ & Tigns on test day \\
& Pen order & $1-8$ & Ohe 8 pens tested \\
& Enrollment cohort & 1 or 2 & Cohort in which each calf was enrolled in the study \\
& Test order & 1 or 2 & Order in which each test (object or stationary human) was performed \\
& Farm & 1 or 2 & Farm of origin \\
& Week & $1-6$ & Age of the calf, in weeks \\
& TEMP score & $0,1,2,3$ & Rectal temperature $\left({ }^{\circ} \mathrm{C}\right)$, categorized into 4 scores: $0=37.8-38.2 ; 1=38.3-38.8 ; 2=$ \\
& & $38.9-39.3 ; 3=239.4$ \\
\hline
\end{tabular}

${ }^{1} \mathrm{BRD}=$ bovine respiratory disease.

${ }^{2}$ Adapted from McGuirk (2008).

missing data for those weeks. Four calves died between wk 1 and 2 test dates and were excluded from all calculations; for these calves, at the time of observation, 2 calves were unable to stand unassisted ( $4 \mathrm{~d}$ old $), 1$ calf had a rectal temperature of $39.7^{\circ} \mathrm{C}(3 \mathrm{~d}$ old $)$, and 1 calf was previously treated by the farm for NCD ( $2 \mathrm{~d}$ old). No necropsies were performed. Final analysis included calves from wk $1(\mathrm{n}=74), 2(\mathrm{n}=64), 3(\mathrm{n}=74), 4(\mathrm{n}$ $=75), 5(\mathrm{n}=75)$, and $6(\mathrm{n}=74)$.

\section{Disease Prevalence and Treatment Incidence}

Prevalence of DIACLIN and BRDCLIN, as identified by research staff, were greatest at wk $2(23.4 \%, 15 / 64)$ and $4(40.0 \%, 30 / 75)$, respectively (Table 3$)$. Disease incidence was calculated based on diagnosis of a disease by either research or farm staff. Fifty-five cases of NCD were identified in 52 calves. A single episode of NCD was identified in $65.3 \%(49 / 75)$ of calves and 2 separate cases were identified in $4 \%(3 / 75)$ of calves. Research staff identified 23 cases of NCD that were not identified by farm staff. The farm staff identified 32 cases of NCD that were not identified by researchers. Five calves were treated by the farm staff for NCD that were not identified by research staff as having DIACLIN on test day.

Eighty-three cases of BRD were identified in 63 calves. A single case of respiratory disease was identified in $58.7 \%(44 / 75)$ of calves, $24 \%(18 / 75)$ of calves were diagnosed with 2 separate cases of respiratory disease, and 1 calf was identified with 3 separate cases of respiratory disease. Fifty-seven percent $(47 / 83)$ of calves identified with BRD by researchers had a BRD

Table 3. Proportion (\%, no./no.) of calves in each disease category on test day, by week

\begin{tabular}{|c|c|c|c|c|c|c|c|}
\hline Week & $\begin{array}{c}\text { Number } \\
\text { tested }\end{array}$ & DIACLIN $^{1}$ & DIAREC $^{2}$ & BRDCLIN $^{3}$ & BRDREC $^{4}$ & Fever $^{5}$ & Umbilical $^{6}$ \\
\hline 1 & 74 & $13.5(10 / 74)$ & $2.7(2 / 74)$ & $5.4(4 / 74)$ & 0 & $21.6(16 / 74)$ & 0 \\
\hline 2 & 64 & $23.4(15 / 64)$ & $34.4(22 / 64)$ & $15.6(10 / 64)$ & $6.3(4 / 64)$ & $17.2(11 / 64)$ & $4.7(3 / 64)$ \\
\hline 4 & 75 & $2.7(2 / 75)$ & $6.7(5 / 75)$ & $40.0(30 / 75)$ & $20.0(15 / 75)$ & $13.3(10 / 75)$ & $5.3(4 / 75)$ \\
\hline 5 & 75 & $1.3(1 / 75)$ & $2.7(2 / 75)$ & $24.0(18 / 75)$ & $34.7(26 / 75)$ & $16.0(12 / 75)$ & $2.7(2 / 75)$ \\
\hline 6 & 74 & $1.4(1 / 74)$ & 0 & $33.8(25 / 74)$ & $23.0(17 / 74)$ & $9.5(7 / 74)$ & $1.4(1 / 74)$ \\
\hline
\end{tabular}

${ }^{1}$ Clinical signs of neonatal calf diarrhea on test day; fecal consistency was extremely watery.

${ }^{2}$ Signs of neonatal calf diarrhea in previous $7 \mathrm{~d}$; no clinical signs on test day.

${ }^{3}$ Bovine respiratory disease (BRD) score of at least 2 categories with a score of 2 or greater.

${ }^{4}$ Signs of BRD in previous $10 \mathrm{~d}$; no clinical signs on test day.

${ }^{5}$ Rectal temperature $\geq 39.4^{\circ} \mathrm{C}$ on test day; fever and disease are not always mutually exclusive.

${ }^{6}$ Infected umbilical (score of 3 or greater). 
Table 4. Proportion (\%, no./no.) of calves that approached novel object by week and disease category

\begin{tabular}{|c|c|c|c|c|c|c|c|}
\hline Week & All calves & DIACLIN $^{1}$ & DIAREC $^{2}$ & BRDCLIN $^{3}$ & BRDREC $^{4}$ & Healthy $^{5}$ & Fever $^{6}$ \\
\hline 2 & $28.1^{\mathrm{b}}(18 / 64)$ & $33.3(5 / 15)$ & $27.3(6 / 22)$ & $40.0(4 / 10)$ & $50.0(2 / 4)$ & $25.0(5 / 20)$ & $27.3(3 / 11)$ \\
\hline 3 & $40.5^{\mathrm{b}}(30 / 74)$ & $60.0(3 / 5)$ & $32.0(8 / 25)$ & $34.8(8 / 23)$ & $42.9(3 / 7)$ & $50.0(12 / 24)$ & $27.3(3 / 11)$ \\
\hline 5 & $49.3^{\mathrm{b}}(37 / 75)$ & $0(0 / 1)$ & $100.0(2 / 2)$ & $18.0(3 / 18)$ & $61.5(16 / 26)$ & $57.1(16 / 28)$ & $16.7(2 / 12)$ \\
\hline 6 & $66.2^{\mathrm{c}}(49 / 74)$ & $100.0(1 / 1)$ & $0 / 0$ & $52.0(13 / 25)$ & $70.6(12 / 17)$ & $73.3(22 / 30)$ & $57.1(4 / 7)$ \\
\hline Total & $39.7(173 / 436)$ & $26.5(9 / 34)$ & $30.4(17 / 56)$ & $36.4(40 / 110)$ & $52.2(36 / 69)$ & $43.3(78 / 180)$ & $22.4(15 / 67)$ \\
\hline
\end{tabular}

${ }^{\mathrm{a}-\mathrm{c}}$ Proportions within a column with different superscripts differ $(P<0.05)$.

${ }^{1}$ Clinical signs of neonatal calf diarrhea on test day; fecal consistency was extremely watery.

${ }^{2}$ Signs of neonatal calf diarrhea in previous $7 \mathrm{~d}$; no clinical signs on test day.

${ }^{3}$ Bovine respiratory disease (BRD) score of at least 2 categories with a score of 2 or greater.

${ }^{4}$ Signs of BRD in previous $10 \mathrm{~d}$; no clinical signs on test day.

${ }^{5}$ Calves on test day that did not have BRDCLIN, BRDREC, DIACLIN, DIACASE, or a fever.

${ }^{6} \mathrm{Rectal}$ temperature $\geq 39.4^{\circ} \mathrm{C}$ on test day; fever and disease are not always mutually exclusive.

score of 5. Research staff identified 6 cases of BRD that were not identified by farm staff. The farm staff identified an additional 22 cases that were not identified by research staff. Overall, 4 calves were treated by the farm staff for BRD that were not identified by research staff as having BRDCLIN on test day. The Se of farm staff for the identification of BRDREC was $25.3 \%$ (21/83). Twenty-four percent (26/110) of instances of BRDCLIN identified by research staff were treated by the farm for BRD in the previous $10 \mathrm{~d}$. The farm treated $50.7(38 / 75), 63.7(47 / 75), 5.3(4 / 75)$, and 5.3 $(4 / 75)$ of calves for BRD, NCD, umbilical infections, and lameness, respectively.

\section{Approach Tests}

During wk 6, the greatest proportion of calves approached, with $66.2(49 / 74)$ and $67.6 \%(50 / 74)$ approaching OBJ and $\mathrm{SH}$, respectively (Tables 4 and 5). Eighty-three (144/173) and $70.6 \%(113 / 160)$ of calves approached during the first $30 \mathrm{~s}$ for OBJ and SH ap- proach tests, respectively. The probability of approach for OBJ and SH were highly associated $(P<0.01)$. Twenty-six percent $(115 / 436)$ of calves approached both tests and 50\% (218/436) did not approach either. Thirteen (58/436) and $10.3 \%(45 / 436)$ of calves only approached the OBJ and $\mathrm{SH}$, respectively.

\section{Effect of Disease}

Novel Object. Overall, 36.4\% (40/110) of calves with BRDCLIN approached OBJ, compared with $43.3 \%(78 / 180)$ of healthy calves (Table 4). Probability of approaching OBJ was significantly affected by BRDCLIN $(P=0.03)$, test order $(P=0.007)$, and week $(P<0.0001)$. Calves with BRDCLIN were 0.5 $(95 \% \mathrm{CI}=0.3-0.9)$ times as likely to approach OBJ compared with calves without BRDCLIN $(P=0.03)$. Calves were $0.4(95 \% \mathrm{CI}=0.2-0.8)$ times as likely to approach OBJ if this test was performed first. The proportion of all calves that approached OBJ increased as calves got older (Table 4$)$. Calves were 40 (95\% CI =

Table 5. Proportion (\%, no./no.) of calves that approached stationary human by week and disease category

\begin{tabular}{llcccccc}
\hline Week & All calves & DIACLIN & DIAREC & BRDCLIN & BRDREC & Healthy $^{5}$ \\
\hline 1 & $17.6^{\mathrm{a}}(13 / 74)$ & $10.0(1 / 10)$ & $0(0 / 2)$ & $0(0 / 4)$ & $0 / 0$ & $21.6(11 / 51)$ & $6.3(1 / 16)$ \\
2 & $18.8^{\mathrm{ab}}(12 / 64)$ & $20.0(3 / 15)$ & $9.1(2 / 22)$ & $20.0(2 / 10)$ & $50.0(2 / 4)$ & $20.0(4 / 20)$ \\
3 & $32.4^{\mathrm{c}}(24 / 74)$ & $20.0(1 / 5)$ & $24.0(6 / 25)$ & $21.7(5 / 23)$ & $42.9(3 / 7)$ & $45.8(11 / 24)$ & $27.3(1 / 11)$ \\
4 & $40.0^{\mathrm{bc}}(30 / 75)$ & $0(0 / 2)$ & $20.0(1 / 5)$ & $36.7(11 / 30)$ & $40.0(6 / 15)$ & $48.2(13 / 27)$ & $20.0(2 / 10)$ \\
5 & $41.3^{\mathrm{c}}(31 / 75)$ & $0(0 / 1)$ & $100.0(2 / 2)$ & $22.2(4 / 18)$ & $50.0(13 / 26)$ & $50.0(14 / 28)$ & $8.3(1 / 12)$ \\
6 & $67.6^{\mathrm{d}}(50 / 74)$ & $100.0(1 / 1)$ & $0 / 0$ & $52.0(13 / 25)$ & $76.5(13 / 17)$ & $73.3(22 / 30)$ & $57.1(4 / 7)$ \\
Total & $36.1(160 / 436)$ & $17.7(6 / 34)$ & $19.6(11 / 56)$ & $31.8(35 / 110)$ & $53.6(37 / 69)$ & $41.7(75 / 180)$ & $17.9(12 / 67)$ \\
\hline
\end{tabular}

${ }^{\mathrm{a}-\mathrm{d}}$ Proportions within a column with different superscripts differ $(P<0.05)$.

${ }^{1}$ Clinical signs of neonatal calf diarrhea on test day; fecal consistency was extremely watery.

${ }^{2}$ Signs of neonatal calf diarrhea in previous $7 \mathrm{~d}$; no clinical signs on test day.

${ }^{3}$ Bovine respiratory disease (BRD) score of at least 2 categories with a score of 2 or greater.

${ }^{4} \mathrm{Signs}$ of BRD in previous $10 \mathrm{~d}$; no clinical signs on test day.

${ }^{5}$ Calves on test day that did not have BRDCLIN, BRDREC, DIACLIN, DIACASE, or a fever.

${ }^{6}$ Rectal temperature $\geq 39.4^{\circ} \mathrm{C}$ on test day; fever and disease are not always mutually exclusive. 
Table 6. Sensitivity and specificity (\%, no./no.) of novel object and stationary human approach tests for the identification of preweaned group-housed calves with BRDCLIN ${ }^{1}$

\begin{tabular}{|c|c|c|c|c|}
\hline \multirow[b]{2}{*}{ Week } & \multicolumn{2}{|c|}{ Novel object } & \multicolumn{2}{|c|}{ Stationary human } \\
\hline & Sensitivity & Specificity & Sensitivity & Specificity \\
\hline 1 & $100.0(4 / 4)$ & $13.7(7 / 51)$ & $100.0(4 / 4)$ & $21.6(11 / 51)$ \\
\hline 2 & $60.0(6 / 10)$ & $25.0(5 / 20)$ & $80.0(8 / 10)$ & $20.0(4 / 20)$ \\
\hline 3 & $65.2(15 / 23)$ & $50.0(12 / 24)$ & $78.3(18 / 23)$ & $45.8(11 / 24)$ \\
\hline 4 & $60.0(18 / 30)$ & $59.3(16 / 27)$ & $63.3(19 / 30)$ & $48.1(13 / 27)$ \\
\hline 5 & $83.3(15 / 18)$ & $57.1(16 / 28)$ & $77.8(14 / 18)$ & $50.0(14 / 28)$ \\
\hline 6 & $48.0(12 / 25)$ & $73.3(22 / 30)$ & $48.0(12 / 25)$ & $73.3(22 / 30)$ \\
\hline Total & $63.6(70 / 110)$ & $43.3(78 / 180)$ & $68.2(75 / 110)$ & $41.7(75 / 180)$ \\
\hline
\end{tabular}

${ }^{1}$ Bovine respiratory disease score of at least 2 categories with a score of 2 or greater on test day.

13.3-125) times more likely to approach OBJ during wk 6 compared with wk $1(P<0.0001)$. The following were not significantly associated with the probability of approach for OBJ: BRDREC, DIACLIN, and DIAREC $(P>0.05)$.

$\boldsymbol{S H}$. Overall, $31.8 \%(35 / 110)$ of calves with BRDCLIN approached SH, compared with 41.7\% (75/180) of healthy calves (Table 5$)$. Calves with clinical signs of BRD $(P=0.02)$, DIAREC $(P=0.03)$, and week $(P=$ $0.03)$ were significantly associated with probability of approach. Calves with BRDCLIN were 0.5 (95\% CI = 0.3-0.9) times as likely to approach SH compared with calves without BRDCLIN. Calves with DIAREC were $0.4(95 \% \mathrm{CI}=0.2-0.9)$ times as likely to approach $\mathrm{SH}$ compared with calves without DIAREC. The proportion of calves that approached increased as calves got older (Table 5). Calves were 14.9 (95\% CI $=5.7-38.5)$ times as likely to approach SH during wk 6 compared with wk $1(P<0.0001)$.

\section{Effect of Rectal Temperature}

There was no difference between the probability of approach between TEMP scores of 0,1 , and $2(P>$ $0.05)$ for OBJ or SH. Therefore, TEMP scores were combined into fever (TEMP score of $3 ; \mathrm{n}=67$ ) and no fever (TEMP scores of 0,1 , and $2 ; \mathrm{n}=369$ ).

Novel Object. Overall, 22.4\% (15/67) of calves with a fever approached OBJ, compared with $43.3 \%(78 / 180)$ of healthy calves (Table 4$)$. Fever $(P<0.01)$, test or$\operatorname{der}(P=0.007)$, and week $(P<0.0001)$ affected the probability of approaching OBJ. Calves with fever were $0.4(95 \%$ CI $=0.2-0.8)$ times less likely to approach OBJ compared with calves without fever. Calves were $2.5(95 \% \mathrm{CI}=1.3-4.7)$ times more likely to approach OBJ if the OBJ test was performed second, rather than first. The proportion of calves that approached OBJ increased with week $(P<0.0001)$.

$\boldsymbol{S H}$. Overall, $17.9 \%(12 / 67)$ of calves with a fever approached SH, compared with $41.7 \%(75 / 180)$ of healthy calves (Table 5). Fever $(P<0.05)$ and week $(P$ $<0.0001)$ affected the probability of approach. Calves with fever were $0.4(95 \% \mathrm{CI}=0.1-0.7)$ times less likely to approach $\mathrm{SH}$ compared with calves without fever. The proportion of calves that approached SH increased with week $(P<0.0001)$.

\section{Se, Sp, and Predictive Values}

Novel Object. The Se and Sp of the OBJ approach test were $63.6(70 / 110)$ and $43.3 \%(78 / 180)$ for BRDCLIN, respectively (Table 6). The PV+ of OBJ for the identification of BRDCLIN was $26.6 \%(70 / 263)$ and PV - was $45.1 \%(78 / 173)$. The Se and Sp of OBJ for identifying a fever were $77.6(52 / 67)$ and $43.3 \%$ $(78 / 180)$, respectively (Table 7$)$. The PV+ of OBJ for the identification of fever was $19.8 \%(52 / 263)$ and PVwas $45.1 \%(78 / 173)$.

$\boldsymbol{S H}$. The Se and Sp of SH for identifying BRDCLIN were $68.2(75 / 110)$ and $41.7 \%(75 / 180)$, respectively (Table 6). The PV+ of SH for the identification of BRDCLIN was $27.1 \%(75 / 276)$ and PV - was $46.9 \%$ (75/160). The Se and Sp of SH for identifying a fever were $82.1(55 / 173)$ and $41.7 \%(78 / 180)$, respectively (Table 7 ). The PV + of SH for the identification of fever was $20.0 \%(55 / 276)$ and PV- was 46.9\% (75/160).

\section{DISCUSSION}

Calves with BRDCLIN were less likely to approach OBJ and SH compared with calves without BRDCLIN. Additionally, calves with a fever were less likely to approach OBJ and SH compared with calves without a fever. Recovering from NCD significantly affected approach for SH. Approaching OBJ and SH likely represented interest in novelty or exploratory behavior. These results support the hypothesis that sickness behavior decreases exploratory behavior (Hart, 1988) and are consistent with the findings of a previous study in which mice showed decreased exploratory behavior 
Table 7. Sensitivity and specificity $(\%$, no./no.) of novel object and stationary human approach tests for the identification of preweaned group-housed calves with fever ${ }^{1}$

\begin{tabular}{llcllc}
\hline & \multicolumn{2}{c}{ Novel object } & & \multicolumn{2}{c}{ Stationary human } \\
\cline { 2 - 3 } \cline { 5 - 6 } Week & Sensitivity & Specificity & & Sensitivity & Specificity \\
\hline 1 & $93.8(15 / 16)$ & $13.7(7 / 51)$ & & $93.8(15 / 16)$ & $21.6(11 / 51)$ \\
2 & $72.7(8 / 11)$ & $25.0(5 / 20)$ & & $90.9(10 / 11)$ & $20.0(4 / 20)$ \\
3 & $72.7(8 / 11)$ & $50.0(12 / 24)$ & & $72.7(8 / 11)$ & $45.8(11 / 24)$ \\
4 & $80.0(8 / 10)$ & $59.3(16 / 27)$ & & $80.0(8 / 10)$ & $48.1(13 / 27)$ \\
5 & $83.3(10 / 12)$ & $57.1(16 / 28)$ & & $91.6(11 / 12)$ & $50.0(14 / 28)$ \\
6 & $42.8(3 / 7)$ & $73.3(22 / 30)$ & & $42.9(3 / 7)$ & $73.3(22 / 30)$ \\
Total & $77.6(52 / 67)$ & $43.3(78 / 180)$ & & $82.1(55 / 67)$ & $41.7(75 / 180)$ \\
\hline
\end{tabular}

${ }^{1}$ Rectal temperature $\geq 39.4^{\circ} \mathrm{C}$ on test day.

toward a novel object when injected with LPS (Haba et al., 2012).

A greater proportion of calves approached the OBJ compared with the SH. Given that farm staff entered the pen on a daily basis, it is possible that calves were more accustomed to the presence of humans and may have been more motivated to explore a novel object in their environment. Alternatively, calves may have been less likely to approach a human who may represent a potential negative stimulus based on previous handling. However, treatment history did not affect approach at the univariable level and thus was not included in multivariable analysis.

The hypothesis that ill calves may be less motivated to perform exploratory behavior, due to the feelings of malaise that accompany illness, was supported by the decreased probability of calves with BRDCLIN to approach during OBJ and SH tests. This decreased exploratory behavior provides further support that illness decreases welfare. Ohl and van der Staay (2012) suggest that the ability of an animal to perform exploration is an important part of animal welfare. Exploratory behavior can be extrinsic, which serves to gather information about food or water, or intrinsic, which is the exploration of stimuli that have no biological significance (Berlyne, 1960; Wood-Gush and Vestergaard, 1989). Calves in the study population were not subjected to pen changes as a standard management practice, nor had they undergone weaning. However, calves that exhibit decreased exploratory behavior due to illness are less able to gain information about their environment. This decreased exploration may result in difficulty adjusting to new experiences, such as pen changes, that take place as management practices on other farms. This may lead to an increased latency to find feed or water, and thus the inability to obtain necessary nutrients. In the present study, calves with BRDCLIN and fever were less likely to engage in exploratory behavior, providing further support that illness decreases welfare.
Recovering from NCD affected the probability of approaching SH. In a previous study, calves altered their feeding behavior for several days after the onset of NCD (Todd et al., 2010), and our findings further support changes in behavior due to illness.

Clinical NCD did not affect the probability of approach for either test. This may be due to the low prevalence of DIACLIN, which would limit the ability to detect a behavioral difference between calves with and without DIACLIN. In addition, research staff identification of this illness may not have been sensitive enough due to the use of spontaneous NCD as an indicator and a short observation period. This lower sensitivity would bias results toward the null. Alternatively, NCD occurred mostly during wk 1 and 2 when relatively few calves approached overall. This may be due to cattle's evolution as a hider species, in which the animals display little activity during the first 2 to $3 \mathrm{wk}$ of life (Fraser and Broom, 1997). Conversely, it is possible that with more frequent approach tests calves would be more curious and, as a result, a greater proportion may approach. More research is necessary to determine the behavior of calves when approach tests are performed more frequently. However, the present study suggests that approach tests that measure exploratory behavior may not be the best way to screen for illness in calves less than 3 wk of age.

Calves' behavior was altered when they exhibited clinical signs of $B R D$, but recovering from $B R D$ (BRDREC) did not affect the behavior. This suggests that approach tests are most useful at identifying calves with clinical signs of BRD; the current study population had a high prevalence of BRD. Lago et al. (2006) reported a mean BRD prevalence of $14 \%$, with ranges from 0 to $37 \%$, but only included individually housed calves in naturally ventilated barns. Also, Lago et al. (2006) used a BRD score of 6 or greater as the criteria for BRD. In the present study, a BRD score of 4 or greater was used as a case definition by research- 
ers for BRD to test the efficacy of approach tests as screening tools. The clustering of at least 2 abnormal clinical signs is indicative of respiratory disease (S. M. McGuirk, University of Wisconsin, Madison, personal communication). This criterion was used to ensure that the calf had BRD rather than another illness that may affect a single health sign. As an effective screening tool, it is desirable to have a test that is highly sensitive to animals that are in the early stages of illness. Due to more sensitive criteria for BRD in the present study, it is possible that true BRD prevalence was overestimated. This would have decreased the magnitude of the difference between calves with and without BRDCLIN, or biased the results toward the null. Additionally, the farm identified 4 calves with BRD and 5 calves with NCD on test day that were not identified by research staff. This may have been due to either misdiagnosis by farm staff, resolution of clinical signs, or the absence of clinical signs when research staff observed the calf. For consistency, the definitions of BRDCLIN and DIACLIN were based on the identification of calves with clinical signs by research staff only. This misclassification may have resulted in a lower disease prevalence and may have biases approach results toward the null. However, this discrepancy on test day included a small number of calves, and so it is unlikely that this would have profoundly affected the results. If a BRD score of 6 or greater was the definition of disease in the present study, the prevalence would be $6.4 \%$, which is within the range of $\mathrm{BRD}$ prevalence reported by Lago et al. (2006).

Generally, a rectal temperature above $39.4^{\circ} \mathrm{C}$ is considered a fever, which corresponds to a TEMP score of 3. Sickness behavior serves to better enable the febrile response, which is thought to be beneficial to fighting infection (Hart, 1988). Fever has a high energetic cost (Kluger, 1991), thus animals alter their behavior to conserve heat and energy, which may include decreased exploratory behavior. This hypothesis is supported by our data, in which calves with a fever were less likely to approach both OBJ and SH compared with calves without a fever. Fever is a component of many different diseases and the ability to identify febrile animals would be beneficial for disease detection.

Age affected the probability of approach for both tests. A greater proportion of calves approached as age increased. Due to disease clustering by age, the interaction between age and health status on probability of approach could not be determined. In this population, calves tended to get sick with NCD earlier in life (highest at wk 2) and with BRD later in life (highest during wk 4). This is consistent with previous findings in which NCD typically affected calves younger than 30 d of age (Virtala et al., 1996b; Svensson et al., 2006;
USDA, 2010) and respiratory disease usually affected calves older than $30 \mathrm{~d}$ of age (Svensson et al., 2006; USDA, 2010). A combination of high Se and Sp for identifying calves with BRDCLIN occurred during later weeks. Consequently, this further supports the argument that approach tests have the greatest value for disease detection when calves are older.

If OBJ was performed second, calves were more likely to approach. This may be because calves were accustomed to farm staff walking in and out of the pens and may not have noticed the placement of the cone in the pen. After a person was in the pen, calves were probably more aware of the environment and thus noticed the cone. Perhaps, if a more noticeable or larger object was used the effect of test order may not be evident.

Sensitivity and Sp were used to evaluate approach tests as screening tools for disease detection. Ideally, a screening test should have a high Se so that a large proportion of animals that are ill can be identified, and a moderate level of $\mathrm{Sp}$ to minimize time examining calves that are false positives. When considering BRDCLIN, the OBJ test had an overall Se and Sp of 63.6 and $43.3 \%$, respectively. The SH test had Se and Sp of 68.2 and $41.7 \%$, respectively, for identifying calves with BRDCLIN. Using farm treatment of clinical cases as a surrogate measure of disease detection, the Se of farm staff for identification of calves with BRDCLIN was $25.3 \%$. Although the Se of both approach tests are lower than what may be ideal for a screening test, they would identify a greater proportion of calves with BRDCLIN compared with the farm staff. Therefore, approach tests may improve the ability of farm staff to detect calves with BRDCLIN. The measurement of farm Se was based on farm treatment records, which represent only those animals the farm deemed severe enough to treat. It is possible that the farm did not see reason to treat some of the calves identified as ill by research staff. For ethical reasons, the health score information was emailed to the farm $24 \mathrm{~h}$ after every test day; calves treated based on findings from research staff may not be an accurate depiction of the farm's ability to detect disease. As such, it is possible that the Se of the farm was overestimated. The predictive values are an indication of the usefulness when a test is applied to animals of unknown disease status and are driven by the true prevalence of disease and the Se and Sp of the test (Dohoo et al., 2003). Both tests resulted in low predictive values that were less than desirable. Both the OBJ and $\mathrm{SH}$ tests had PV+ values of about $27 \%$ for the identification of BRDCLIN, which would mean that $27 \%$ of the calves that tested positive (i.e., the calves did not approach) would actually have BRDCLIN. This would require farm staff to spend time examining animals that are not actually 
sick. The PV - for the identification of BRDCLIN were 45.1 and $46.9 \%$ for the OBJ and SH tests, respectively. This means that 45.1 or $46.9 \%$ of animals that tested negative (i.e., the calves approached) would actually be healthy. This would mean that approximately half of calves that tested negative were actually sick, and thus would not be identified by farm staff. Whereas the $\mathrm{PV}-$ and PV+ are not ideal, the approach tests still have merit because they are more sensitive for disease identification than farm staff and they require a certain amount of time to spend observing calves, which may allow farm staff to identify sick animals through other observations.

The overall Se of OBJ for identifying febrile calves was $77.6 \%$ and the Sp was $43.3 \%$. The overall Se of $\mathrm{SH}$ for identifying calves with fever was $82.1 \%$, which was greater than the Se of the OBJ test, but $\mathrm{Sp}$ of $\mathrm{SH}$ remained similar at $41.7 \%$. Because the combination of high Se and moderate Sp was not obtained, an approach test should not be the sole screening method for illness in calves, although it is quick and inexpensive and therefore has merit as a supplemental screening tool. An additional benefit is that it requires a set period of dedicated time in which calves must be observed. It is possible that, when combined with other behavioral measures of illness, the Se and Sp of this test may be improved.

Different populations of calves should also be examined given that social factors can influence exploratory behavior (Boissy and Le Neindre, 1990). Before approach tests are implemented on farm, research is needed to determine how calves behave if approach tests are used on a regular basis. It is possible that calves may become habituated to an approach test over time and therefore would not be motivated to explore. Older cattle show decreased interaction with a novel object when exposed more than once to the same object (Kilgour et al., 2006). When using approach tests as a disease detection tool, this may be remedied by using different objects that would be novel and therefore may facilitate more exploratory behavior. However, it would also be useful to compare different types of approach tests among calves of similar ages to determine if calves become habituated or if they will continue to engage in exploratory behavior. In the present study, the probability of approach increased with age; however, it is unknown how different methods of approach tests would compare when age is controlled for. Also, the space allowance per calf in the present study was similar to that of an experimental pen in previous work which found that, compared with larger space allowances, calves performed less synchronous lying but agonistic behavior and social play was not affected by space allowance (Faerevik et al., 2008). Therefore, it is unlikely that stocking density influenced the probability of approach in the present study. Behavioral measures of illness that can be easily observed in a farm setting would be a beneficial tool for farm staff as it would provide a clearer definition of the appearance of a sick calf, and may improve the ability to detect disease. The integration of multiple behavioral measures may be used to improve illness detection.

\section{CONCLUSIONS}

Calves with BRDCLIN were less likely to approach OBJ and SH compared with calves without BRDCLIN. Calves with a fever were less likely to approach OBJ and SH compared with calves without a fever. These findings support the hypothesis that calves with clinical signs of BRD and febrile calves exhibit decreased exploratory behavior. Whereas Se, Sp, PV+, and PVare less than desirable, and do not allow the authors to recommend approach tests as a sole method for disease identification, the OBJ and SH approach tests can be a useful tool to detect BRDCLIN and fever in calves over 3 wk of age. Sickness behavior in a socially competitive environment should be further studied to improve disease detection in group-housed calves.

\section{ACKNOWLEDGMENTS}

This material is based upon work supported by the National Institute of Food and Agriculture, USDA (Washington, DC), under ID number WIS01685. Any opinions, findings, conclusions, or recommendations expressed in this publication are those of the author(s) and do not necessarily reflect the view of the USDA.

\section{REFERENCES}

Anderson, N. 2013. Group Housing and Feeding of Milk-Fed Calves: Moving Closer to Nature's Way. OMAFRA, Ontario, Canada.

Bach, A. 2011. Associations between several aspects of heifer development and dairy cow survivability to second lactation. J. Dairy Sci. 94:1052-1057.

Berlyne, D. E. 1960. Conflict, Arousal, and Curiosity. McGraw Hill, New York, NY.

Boissy, A., and P. Le Neindre. 1990. Social influences on the reactivity of heifers: Implications for learning abilities in operant conditioning. Appl. Anim. Behav. Sci. 25:149-165.

Borderas, T. F., J. Rushen, M. A. G. von Keyserlingk, and A. M. B. de Passillé. 2009. Automated measurement of changes in feeding behavior of milk-fed calves associated with illness. J. Dairy Sci. 92:4549-4554.

Dohoo, I., W. Martin, and H. Stryhn. 2003. Veterinary Epidemiologic Research. AVC Inc., University of Prince Edward Island, Charlottetown, Prince Edward Island, Canada.

Faerevik, G., K. Tjentland, S. Lovik, I. L. Andersen, and K. E. Boe. 2008. Resting pattern and social behaviour of dairy calves housed in pens with different sized lying areas. Appl. Anim. Behav. Sci. 114:54-64. 
Fraser, A. F., and D. M. Broom. 1997. Farm Animal Behaviour and Welfare. CABI, New York, NY.

Gibbons, J., A. Lawrence, and M. Haskell. 2009. Responsiveness of dairy cows to human approach and novel stimuli. Appl. Anim. Behav. Sci. 116:163-173.

Haba, R., N. Shintani, Y. Onaka, H. Wang, R. Takenaga, A. Hayata, A. Baba, and H. Hashimoto. 2012. Lipopolysaccharide affects exploratory behaviors toward novel objects by impairing cognition and/or motivation in mice: Possible role of activation of the central amygdala. Behav. Brain Res. 228:423-431.

Hart, B. L. 1988. Biological basis of the behavior of sick animals. Neurosci. Biobehav. Rev. 12:123-137.

Hultgren, J., C. Svensson, D. O. Maizon, and P. A. Oltenacu. 2008 Rearing conditions, morbidity and breeding performance in dairy heifers in southwest Sweden. Prev. Vet. Med. 87:244-260.

Kilgour, R. J., G. J. Melville, and P. L. Greenwood. 2006. Individual differences in the reaction of beef cattle to situations involving social isolation, close proximity of humans, restraint and novelty. Appl. Anim. Behav. Sci. 99:21-40.

Kluger, M. J. 1991. Fever: role of pyrogens and cryogens. Physiol. Rev. 71:93-127.

Lago, A., S. M. McGuirk, T. B. Bennett, N. B. Cook, and K. V Nordlund. 2006. Calf respiratory disease and pen microenvironments in naturally ventilated calf barns in winter. J. Dairy Sci. 89:4014-4025.

Lauber, M. C. Y., P. H. Hemsworth, and J. L. Barnett. 2006. The effects of age and experience on behavioural development in dairy calves. Appl. Anim. Behav. Sci. 99:41-52.

McGuirk, S. M. 2008. Disease management of dairy calves and heifers. Vet. Clin. North Am. Food Anim. Pract. 24:139-153.

Millman, S. T. 2007. Sickness behaviour and its relevance to animal welfare assessment at the group level. Anim. Welf. 16:123-125.

Murphy, L. B., and D. G. M. Wood-Gush. 1978. The interpretation of the behavior of domestic fowl in strange environments. Biol. Behav. 3:39-61.

Ohl, F., and F. J. van der Staay. 2012. Animal welfare: At the interface between science and society. Vet. J. 192:13-19.

Quimby, W. F., B. F. Sowell, J. G. P. Bowman, M. E. Branine, M. E. Hubbert, and H. W. Sherwood. 2001. Application of feeding behaviour to predict morbidity of newly received calves in a commercial feedlot. Can. J. Anim. Sci. 81:315-320.
Schaefer, A. L., N. J. Cook, C. Bench, J. B. Chabot, J. Colyn, T. Liu, E. K. Okine, M. Stewart, and J. R. Webster. 2012. The noninvasive and automated detection of bovine respiratory disease onset in receiver calves using infrared thermography. Res. Vet. Sci. 93:928-935.

Sivula, N. J., T. R. Ames, W. E. Marsh, and R. E. Werdin. 1996. Descriptive epidemiology of morbidity and mortality in Minnesota dairy heifer calves. Prev. Vet. Med. 27:155-171.

Stanton, A. L., D. F. Kelton, S. J. LeBlanc, J. Wormuth, and K. F. Leslie. 2012. The effect of respiratory disease and a preventative antibiotic treatment on growth, survival, age at first calving, and milk production of dairy heifers. J. Dairy Sci. 95:4950-4960.

Svensson, C., and M. B. Jensen. 2007. Short communication: Identification of diseased calves by use of data from automatic milk feeders. J. Dairy Sci. 90:994-997.

Svensson, C., A. Linder, and S. O. Olsson. 2006. Mortality in Swedish dairy calves and replacement heifers. J. Dairy Sci. 89:4769-4777.

Todd, C. G., S. T. Millman, D. R. McKnight, T. F. Duffield, and K. E. Leslie. 2010. Nonsteroidal anti-inflammatory drug therapy for neonatal calf diarrhea complex: Effects on calf performance. J. Anim. Sci. 88:2019-2028.

USDA. 2010. Heifer Calf Health and Management Practices on U.S. Dairy Operations, 2007. USDA:APHIS:VS, CEAH, Fort Collins, CO.

Virtala, A. M. K., G. D. Mechor, Y. T. Grohn, and H. N. Erb. 1996a The effect of calfhood diseases on growth of female dairy calves during the first 3 months of life in New York state. J. Dairy Sci 79:1040-1049

Virtala, A. M. K., G. D. Mechor, Y. T. Grohn, and H. N. Erb. 1996b. Morbidity from nonrespiratory diseases and mortality in dairy heifers during the first three months of life. J. Am. Vet. Med. Assoc. 208:2043-2046.

Waltner-Toews, D., S. W. Martin, and A. H. Meek. 1986. The effect of early calfhood health status on surviviorship and age at first calving. Can. J. Vet. Res. 50:314-317.

Windeyer, M. C., K. E. Leslie, S. M. Godden, D. C. Hodgins, K. D. Lissemore, and S. J. LeBlanc. 2014. Factors associated with morbidity, mortality, and growth of dairy heifer calves up to 3 months of age. Prev. Vet. Med. 113:231-240.

Wood-Gush, D., and K. Vestergaard. 1989. Exploratory behavior and the welfare of intensively kept animals. J. Agric. Ethics 2:161-169. 